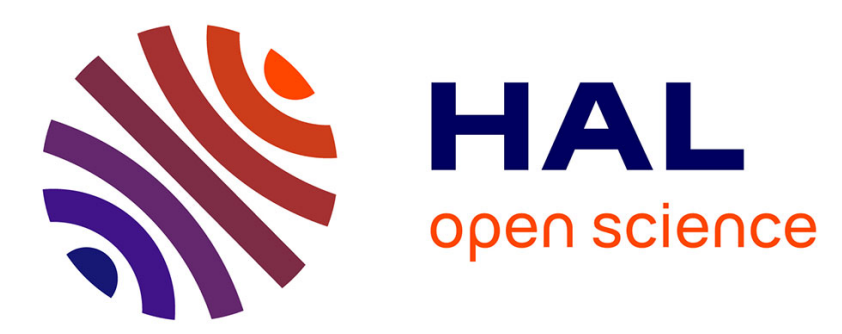

\title{
State-Dependent Sampling for Perturbed Time-Delay Systems
}

Christophe Fiter, Laurentiu Hetel, Wilfrid Perruquetti, Jean-Pierre Richard

\section{To cite this version:}

Christophe Fiter, Laurentiu Hetel, Wilfrid Perruquetti, Jean-Pierre Richard. State-Dependent Sampling for Perturbed Time-Delay Systems. 51st IEEE Conference on Decision and Control, Dec 2012, Maui, Hawaï, United States. pp.CD. hal-00728437

\section{HAL Id: hal-00728437 \\ https://hal.science/hal-00728437}

Submitted on 6 Sep 2012

HAL is a multi-disciplinary open access archive for the deposit and dissemination of scientific research documents, whether they are published or not. The documents may come from teaching and research institutions in France or abroad, or from public or private research centers.
L'archive ouverte pluridisciplinaire HAL, est destinée au dépôt et à la diffusion de documents scientifiques de niveau recherche, publiés ou non, émanant des établissements d'enseignement et de recherche français ou étrangers, des laboratoires publics ou privés. 


\title{
State-Dependent Sampling for Perturbed Time-Delay Systems
}

\author{
Christophe Fiter, Laurentiu Hetel, Wilfrid Perruquetti, and Jean-Pierre Richard
}

\begin{abstract}
In this work we present a state-dependent sampling control that allows for enlarging the sampling intervals of state-feedback control. We consider the case of perturbed linear time-invariant systems with input-delay and guarantee their $\mathcal{L}_{2}$-stability. The approach is based on a novel class of switched Lyapunov-Krasovskii functionals with state-dependent matrices. It results in an LMI problem that allows for enlarging the sampling interval according to the system state. Then, a mapping of the state space is designed offline: it computes for each state of the state space a lower-bound estimation of the maximum allowable sampling interval, which makes it possible to reduce the number of actuations during the real-time control of the system.
\end{abstract}

\section{INTRODUCTION}

Networked Control Systems are often required to share a limited amount of resources, which leads to delays, and to fluctuations of the sampling interval. From the control theory point of view, these phenomena bring up new challenges.

Several studies already questioned the robustness aspect for systems with time-varying sampling ([5], [6], [15], [9]), time-varying delays ([8], [14]), or both time-varying sampling and delays ([10], [11]).

Recently, intensive research has also been conducted to adapt dynamically the sampling so as to reduce the processor and/or network loads while ensuring the desired control performances. There are two main approaches in the literature: "Event-triggered control" ([16], [12]), in which sensors are equipped with special intelligence so that information is sent to the controller only when special events occur (e.g. crossing a frontier of the state space, or a level of a Lyapunov function). The main drawback is that it requires dedicated hardware to continuously monitor the plant state and check the defined stability conditions.

"Self-triggered control" ([17], [13]), which consists in computing at each sampling instant a lower-bound estimation of the next largest admissible sampling interval, so as to emulate

This work was supported by Ministry of Higher Education and Research, Nord-Pas de Calais Regional Council and FEDER through the 'Contrat de Projet Etat Region (CPER) 2007-2013'

The research leading to these results has received funding from the European Community's Seventh Framework Programme (FP7/2007-2013) under grant agreement $n^{0}$ 257462: HYCON2 Network of Excellence "HighlyComplex and Networked Control Systems".

This work was supported by the INTERREG IV A 2 Mers Seas Zeen Cross-border Cooperation Program 2007-2013 under SYSIASS 6-20 project.

The authors are with Laboratoire d'Automatique, Génie Informatique et Signal (CNRS UMR 8219), École Centrale de Lille, 59651 Villeneuve d'Ascq, France - W. Perruquetti and J-.P Richard are with the Non-A Project team, INRIA Lille-Nord Europe, France christophe.fiterecentraliens-lille.org, laurentiu.heteldec-lille.fr, wilfrid.perruquettieinria.fr, jean-pierre.richardeec-lille.fr event-triggered control without resorting to extra hardware. In these works, the computations for the next sampling times are made online, which increases the processor load. Also, it generally stays open to compute a Lyapunov function optimizing the sampling intervals while taking into account the perturbations and the delays.

More recently, in [2], we proposed a third dynamic sampling control approach, "state-dependent sampling", which consists in designing a map of the maximal allowable sampling intervals over conic regions of the state-space, thanks to Linear Matrix Inequalities (LMIs). The advantages are: - the state-dependent sampling map is designed offline (once for all), which allows for reducing the number of online computations compared to self-triggered control;

- the Lyapunov function is optimized so as to enlarge the lower-bound of the sampling map.

However, up to now, only ideal Linear Time-Invariant (LTI) systems were considered.

In the present work, we propose a novel state-dependent sampling approach that guarantees $\mathcal{L}_{2}$-stability for LTI systems subject to both perturbations and input-delay. This approach is based on a new class of Lyapunov-Krasovskii functionals $(L K F)$ with matrices that will switch according to the conic region the sampled-state belongs to. Along with the benefits of the state-dependent sampling approach previously mentioned, the advantages are:

- the proposed LKF takes into account both the perturbations, the delays and the sampling;

- the LKF matrices are computed so as to enlarge the sampling map over the conic regions of the state-space.

The paper is organized as follows: Section II formulates the problem; Section III presents the stability results. Section IV provides the offline algorithm that builds the statedependent sampling map. Section V shows some simulation results, and Section VI sums up the conclusions.

Notations: $M^{T}$ stands for the transpose of $M \in \mathcal{M}_{n, m}$ (the set of real $n \times m$ matrices). $S_{n}$ denotes the set of $n \times n$ symmetric matrices, and $S_{n}^{+}$(resp. $\left.S_{n}^{+*}\right)$ the set of positive (resp. positive definite) matrices $P \succeq 0$ (resp. $P \succ 0$ ) in $S_{n}$. $\mathcal{L}_{2}$ is the space of square-integrable functions on $\mathbb{R}^{+} .\|\cdot\|_{2}$ is the Euclidian norm on $\mathbb{R}^{n}$ and $\|.\|_{\mathcal{L}_{2}}$ is the $\mathcal{L}_{2}$-norm on $\mathcal{L}_{2}$ : $\|f\|_{\mathcal{L}_{2}}=\left(\int_{0}^{\infty}\|f(t)\|_{2}^{2} d t\right)^{\frac{1}{2}}$. For a given maximum delay $h_{2}$, $x_{t}\left(\right.$ resp. $\left.\dot{x}_{t}\right)$ denotes a function such that $x_{t}(\theta)=x(t+\theta)$ (resp. $\dot{x}_{t}(\theta)=\dot{x}(t+\theta)$ ) for all $\theta \in\left[-h_{2}, 0\right]$.

\section{PROBLEM FORMULATION}

We consider the LTI system

$$
\left.\begin{array}{l}
\dot{x}(t)=A x(t)+B u(t)+E w(t) \\
z(t)=C x(t)+D u(t)
\end{array}\right\}, \forall t \geq 0
$$


where $x(t) \in \mathbb{R}^{n}$ is the state vector, $w(t) \in \mathbb{R}^{n_{w}}$ is an exogenous disturbance in $\mathcal{L}_{2}, u(t) \in \mathbb{R}^{n_{u}}$ is the control input, and $z(t) \in \mathbb{R}^{n_{z}}$ is the controlled output. $A, B, C$, $D$, and $E$ are constant matrices of appropriate dimensions.

The feedback control law is defined as:

$$
u(t)=-K x\left(s_{k}\right), \forall t \in\left[t_{k}, t_{k+1}\right),
$$

with a constant feedback matrix gain $K, s_{k}$ the $k^{\text {th }}$ sampling time (when the $k^{\text {th }}$ input is computed) and $t_{k}$ the $k^{\text {th }}$ actuation time (when the $k^{\text {th }}$ computed input is received by the actuators).

The sampling law is defined as

$$
s_{k+1}-s_{k} \equiv \tau_{k} \in\left[\tau^{-}, \tau_{\max }\left(x\left(s_{k}\right)\right)\right],
$$

with a given minimal sampling interval $\tau^{-}>0$ and a maximal state-dependent sampling map $\tau_{\max }: \mathbb{R}^{n} \rightarrow \mathbb{R}^{+}$.

We set ourself in the same context as in [2]: we assume that the state space is covered by a set of $q$ conic regions

$$
\mathcal{R}_{\sigma}=\left\{x \in \mathbb{R}^{n}, x^{T} \Psi_{\sigma} x \geq 0\right\}, \Psi_{\sigma} \in S_{n}, \sigma \in\{1, \cdots, q\},
$$

for which maximal sampling intervals $\tau_{\sigma}^{+} \geq \tau^{-}$are defined. With this assumption, the state-dependent sampling map is considered to be a function of the form

$$
\tau_{\max }(x)=\max _{\sigma \in\{1, \cdots, q\}}\left\{\tau_{\sigma}^{+} \mid x \in \mathcal{R}_{\sigma}\right\}, \forall x \in \mathbb{R}^{n} .
$$

The motivation for studying this class of systems (as well as methods to design these conic regions) has been given in [2], where it is shown that such a description may be used for approximating a self-triggered control scheme. This formulation also proved to be efficient to design the sampling map offline, thus allowing to reduce the number of online computations.

The sampling and actuation times are linked by the relation

$$
s_{k}=t_{k}-h\left(t_{k}\right),
$$

with a delay $h(t)$ assumed to satisfy:

$$
\forall t \geq 0, h(t) \in\left[h_{1}, h_{2}\right], \text { and } \dot{h}(t) \in\left[e_{1}, e_{2}\right],
$$

for given scalars $0 \leq h_{1} \leq h_{2}$ and $e_{1} \leq e_{2}<1$. Since $s_{k+1}-s_{k}>0$, it implies that $t_{k+1}-t_{k} \geq \frac{s_{k+1}-s_{k}}{1-e_{1}}>0$, due to $e_{1}<1$ and thus the control inputs are received by the actuator in the same order as they are sent.

The closed-loop system $\{(1), \cdots,(7)\}$ is denoted as $\mathbf{T}$.

Due to the unknown exogenous disturbances, the system $\mathbf{T}$ will be studied from the $\mathcal{L}_{2}$-stability point of view.

Definition 1: [17] A linear system $\mathbf{F}$ is said to be finitegain $\mathcal{L}_{2}$-stable from $w$ to $\mathbf{F} w$ with an induced gain less than $\gamma$ if $\mathbf{F}$ is a linear operator from $\mathcal{L}_{2}$ into $\mathcal{L}_{2}$ and there exist positive real constants $\gamma$ and $\xi$ such that for all $w \in \mathcal{L}_{2}$,

$$
\|\mathbf{F} w\|_{\mathcal{L}_{2}} \leq \gamma\|w\|_{\mathcal{L}_{2}}+\xi
$$

The present work aims at designing (offline) a statedependent sampling map $\tau_{\max }$ (as defined in (5)) that allows to enlarge the sampling intervals (3) while ensuring the finitegain $\mathcal{L}_{2}$-stability of $\mathbf{T}$ from $w$ to $z$, with a gain less than a fixed $\gamma \geq 0$.

\section{MAIN $\mathcal{L}_{2}$-STABILITY RESULTS}

In this section, we provide $\mathcal{L}_{2}$-stability conditions for the perturbed and delayed system $\mathbf{T}$ for a given maximal statedependent sampling map (5). They are based on a quite general class of LKF (with state-dependent matrices) which take into account delays, perturbations and sampling.

\section{A. Non-delayed case}

In order to simplify the reading, we first consider a simplified version of the system (without delay: $s_{k}=t_{k}$ ), and present the main stability tools, as well as a simplified version of the proposed LKF,

$$
\begin{aligned}
V_{\sigma_{k}}\left(t, x(t), \dot{x}_{t}\right) & =x^{T}(t) P x(t) \\
& +\left(s_{k+1}-t\right) \int_{s_{k}}^{t} \dot{x}^{T}(s) U_{\sigma_{k}} \dot{x}(s) d s
\end{aligned}
$$

defined for all $t \in\left[s_{k}, s_{k+1}\right)$ and $k \in \mathbb{N}$, with matrices $P$ and $U_{\sigma}$ of appropriate dimensions. Parameter $\sigma_{k}$ can be any element $\sigma \in\{1, \cdots, q\}$ such that $x\left(s_{k}\right) \in \mathcal{R}_{\sigma}$ and $\tau^{-} \leq \tau_{k} \leq \tau_{\sigma}^{+}$(there exists at least one, according to (3) and (5)).

The new aspect of the LKF (9) compared to previous works on systems with time-varying samplings ([6], [15], [10]) is the fact that it involves elements that are switching according to the system state. Indeed, note that the matrix term $U_{\sigma_{k}}$ is switching at times $s_{k}$ according to the region the sampled state $x\left(s_{k}\right)$ belongs to (since the switching law $\sigma_{k}$ satisfies $x\left(s_{k}\right) \in \mathcal{R}_{\sigma_{k}}$ ). This statedependent switch is possible thanks to the fact that the functional $V_{\sigma_{k}}$ is continuous at times $s_{k}: V_{\sigma_{k}}\left(s_{k}, x\left(s_{k}\right), \dot{x}_{s_{k}}\right)=$ $\lim _{t \rightarrow s_{k}^{-}} V_{\sigma_{k-1}}\left(t, x(t), \dot{x}_{t}\right)=x^{T}\left(s_{k}\right) \operatorname{Px}\left(s_{k}\right)$.

This new type of switched LKF is well adapted to the stability analysis of systems with state-dependent sampling, but it also provides some advantages regarding the stability analysis of systems with (state-independent) time-varying sampling, as it will be shown in the Example 2 of the Numerical Examples Section.

In the following, as in the framework of [6], we denote

$$
\bar{V}(t)=V_{\sigma_{k}}\left(t, x(t), \dot{x}_{t}\right) \text {, for all } t \in\left[s_{k}, s_{k+1}\right), k \in \mathbb{N} \text {. }
$$

In order to guarantee the $\mathcal{L}_{2}$-stability of the non-delayed system $\mathbf{T}$, we need to provide conditions that ensure that $\bar{V}$ satisfies the conditions of Lemma 4, in the Appendix.

To begin with, we can see that $\bar{V}$ is continuous over $\mathbb{R}^{+}$ (we have shown earlier that it is continuous at times $s_{k}$ ) and differentiable over $\mathbb{R}^{+} \backslash\left\{s_{k}, k \in \mathbb{N}\right\}$. To ensure that $\bar{V}$ is positive definite, we assume that the matrices $P$ and $U_{\sigma}$ are such that $P=P^{T} \succ 0$ and $U_{\sigma}=U_{\sigma}^{T} \succeq 0$, for all $\sigma \in$ $\{1, \cdots, q\}$. Now, all that is needed to ensure the system's $\mathcal{L}_{2}$-stability, is to provide conditions to satisfy

$$
\forall t \neq s_{k}, \quad \dot{\bar{V}}(t)+z^{T}(t) z(t)-\gamma^{2} w^{T}(t) w(t) \leq 0 .
$$

In order to analyse this condition, we study the restriction of $\dot{\bar{V}}$ on any interval $\left[s_{k}, s_{k+1}\right), k \in \mathbb{N}$. First, we compute:

$$
\begin{aligned}
\dot{\bar{V}}(t)= & 2 \dot{x}^{T}(t) P x(t)+\left(s_{k+1}-t\right) \dot{x}^{T}(t) U_{\sigma_{k}} \dot{x}(t) \\
& -\int_{s_{k}}^{t} \dot{x}^{T}(s) U_{\sigma_{k}} \dot{x}(s) d s .
\end{aligned}
$$


Then, using the Jensen inequality [8], we compute an upper-bound of the integral term:

$$
-\int_{s_{k}}^{t} \dot{x}^{T}(s) U_{\sigma_{k}} \dot{x}(s) d s \leq-\left(t-s_{k}\right) \nu^{T}(t) U_{\sigma_{k}} \nu(t),
$$

with

$$
\nu(t)=\frac{1}{t-s_{k}} \int_{s_{k}}^{t} \dot{x}(s) d s=\frac{x(t)-x\left(s_{k}\right)}{t-s_{k}},
$$

and obtain

$$
\begin{aligned}
\dot{\bar{V}}(t) \leq & 2 \dot{x}^{T}(t) P x(t)+\left(s_{k+1}-t\right) \dot{x}^{T}(t) U_{\sigma_{k}} \dot{x}(t) \\
& -\left(t-s_{k}\right) \nu^{T}(t) U_{\sigma_{k}} \nu(t)
\end{aligned}
$$

$\nu(t)$ is well defined by continuity in $t=s_{k}$, since when $t \rightarrow s_{k}, \nu(t) \rightarrow \dot{x}\left(s_{k}\right)$.

We introduce the augmented state vector $\phi(t) \in \mathbb{R}^{3 n+n_{w}}$ :

$$
\phi(t)^{T}=\left[x^{T}(t), x^{T}\left(s_{k}\right), \nu^{T}(t), w^{T}(t)\right],
$$

and matrices $M_{i}$ and $N_{j}$ such that

$$
\begin{aligned}
& x(t)=M_{1} \phi(t), x\left(s_{k}\right)=M_{2} \phi(t), \\
& \nu(t)=M_{3} \phi(t), w(t)=M_{4} \phi(t), \\
& \dot{x}(t)=\left(A M_{1}-B K M_{2}+E M_{4}\right) \phi(t)=N_{1} \phi(t), \\
& z(t)=\left(C M_{1}-D K M_{2}\right) \phi(t)=N_{2} \phi(t) .
\end{aligned}
$$

Using these notations we can rewrite the inequality (14) as

$$
\begin{aligned}
& \dot{\bar{V}}(t)+z^{T}(t) z(t)-\gamma^{2} w^{T}(t) w(t) \leq \\
& \phi^{T}(t)\left[N_{1}^{T} P M_{1}+M_{1}^{T} P N_{1}+\left(s_{k+1}-t\right) N_{1}^{T} U_{\sigma_{k}} N_{1}\right. \\
& \left.-\left(t-s_{k}\right) M_{3}^{T} U_{\sigma_{k}} M_{3}+N_{2}^{T} N_{2}-\gamma^{2} M_{4}^{T} M_{4}\right] \phi(t) .
\end{aligned}
$$

The relation (13) between $\nu(t), x(t)$, and $x\left(s_{k}\right)$ can be written as $H(t) \phi(t)=0$ with $H(t)=\left(t-s_{k}\right) M_{3}-M_{1}+$ $M_{2}$. Therefore, by applying the Finsler's lemma [1] one can include this relation in (17) and obtain that for any matrices $Y_{\sigma_{k}} \in \mathcal{M}_{3 n+n_{w}, n}$ :

$$
\begin{aligned}
& \dot{\bar{V}}(t)+z^{T}(t) z(t)-\gamma^{2} w^{T}(t) w(t) \leq \\
& \phi^{T}(t)\left[N_{1}^{T} P M_{1}+M_{1}^{T} P N_{1}+\left(s_{k+1}-t\right) N_{1}^{T} U_{\sigma_{k}} N_{1}\right. \\
& -\left(t-s_{k}\right) M_{3}^{T} U_{\sigma_{k}} M_{3}+N_{2}^{T} N_{2}-\gamma^{2} M_{4}^{T} M_{4} \\
& +Y_{\sigma_{k}}\left(\left(t-s_{k}\right) M_{3}-M_{1}+M_{2}\right) \\
& \left.+\left(\left(t-s_{k}\right) M_{3}-M_{1}+M_{2}\right)^{T} Y_{\sigma_{k}}^{T}\right] \phi(t) .
\end{aligned}
$$

Since (18) is linear in the variable $t$, it is possible to reduce the number of conditions to be checked by applying Lemma 5 (Appendix) with the variable $\lambda=t \in\left[s_{k}, s_{k+1}\right]$. The two obtained inequalities are both linear in the variable $s_{k+1}-s_{k}$. Thus we can use once again Lemma 5 with the variable $\lambda=s_{k+1}-s_{k} \in\left[\tau^{-}, \tau_{\sigma_{k}}^{+}\right]$to prove that if the 4 inequalities $\xi^{T} \Xi_{i, j \sigma_{k}} \xi \leq 0$ are satisfied for all $\xi \in \mathbb{R}^{3 n+n_{w}}$, with $\Xi_{i, j, \sigma_{k}}$ defined as

$$
\begin{gathered}
\Xi_{i, 1, \sigma}=\Xi_{\sigma}+T_{i, \sigma} N_{1}^{T} U_{\sigma} N_{1} \\
\Xi_{i, 2, \sigma}=\Xi_{\sigma}+T_{i, \sigma}\left[-M_{3}^{T} U_{\sigma} M_{3}+Y_{\sigma} M_{3}+M_{3}^{T} Y_{\sigma}^{T}\right] \\
\Xi_{\sigma}=N_{1}^{T} P M_{1}+M_{1}^{T} P N_{1}+N_{2}^{T} N_{2}-\gamma^{2} M_{4}^{T} M_{4} \\
+Y_{\sigma}\left(-M_{1}+M_{2}\right)+\left(-M_{1}+M_{2}\right)^{T} Y_{\sigma}^{T}
\end{gathered}
$$

with

$$
T_{1, \sigma}=T_{1}=\tau^{-} \text {and } T_{2, \sigma}=\tau_{\sigma}^{+},
$$

then $\dot{\bar{V}}(t)+z^{T}(t) z(t)-\gamma^{2} w^{T}(t) w(t) \leq 0$ for all $t \in$ $\left[s_{k}, s_{k+1}\right)$.

Since we know that $x\left(s_{k}\right) \in \mathcal{R}_{\sigma_{k}}$ (i.e. we have $x^{T}\left(s_{k}\right) \Psi_{\sigma_{k}} x\left(s_{k}\right) \geq 0$ ), we can use the lossless version of the $\mathrm{S}$-procedure [1] on each of the 4 obtained inequalities to show that, if there are scalars $\varepsilon_{i, j, \sigma} \geq 0$ such that the LMIs

$$
\Xi_{i, j, \sigma}+\varepsilon_{i, j, \sigma} M_{2}^{T} \Psi_{\sigma} M_{2} \preceq 0,
$$

hold for $\sigma=\sigma_{k}$, then condition (10) is satisfied. Therefore, we have the following property:

Proposition 1: Consider scalars $\gamma \geq 0$ and $\tau^{-}$, and a set of $q$ conic regions covering the state space $\mathcal{R}_{\sigma}=$ $\left\{x, x^{T} \Psi_{\sigma} x \geq 0\right\}, \Psi_{\sigma} \in S_{n}, \sigma \in\{1, \cdots, q\}$, with maximal sampling intervals $\tau_{\sigma}^{+}$.

The perturbed non-delayed sampled-data system $\mathbf{T}$ is finitegain $\mathcal{L}_{2}$-stable from $w$ to $z$ with a gain less than $\gamma$ if there exist matrices $P \in S_{n}^{+*}, U_{\sigma} \in S_{n}^{+}, Y_{\sigma} \in \mathcal{M}_{3 n+n_{w}, n}$, and scalars $\varepsilon_{i, j, \sigma} \geq 0$ such that (23) is satisfied for all $\sigma \in\{1, \cdots, q\}$ and $(i, j) \in\{1,2\}^{2}$.

\section{B. General delayed case}

Now, we consider the more general system with delay, as well as the switched LKF:

$$
V_{\sigma_{k}}\left(t, x_{t}, \dot{x}_{t}\right)=V_{1}\left(t, x_{t}, \dot{x}_{t}\right)+V_{2, \sigma_{k}}\left(t, x_{t}, \dot{x}_{t}\right),
$$

defined for all $t \in\left[t_{k}, t_{k+1}\right)$ and $k \in \mathbb{N}$, with

$$
\begin{aligned}
V_{1}\left(t, x_{t},\right. & \left.\dot{x}_{t}\right)=\eta^{T}(t) P \eta(t)+\int_{t-h_{1}}^{t} x^{T}(s) Q_{1} x(s) d s \\
& +\int_{t-h(t)}^{t-h_{1}} x^{T}(s) Q_{2} x(s) d s \\
& +\int_{t-h(t)}^{t-h(t)} x^{T}(s) Q_{3} x(s) d s \\
& +\int_{t-h(t)}^{t} \dot{x}^{T}(s)\left(R_{1}+(h(t)-t+s) R_{2}\right) \dot{x}(s) d s \\
& +\int_{-h_{2}}^{0} \int_{t+\theta}^{t} \dot{x}^{T}(s) R_{3} \dot{x}(s) d s d \theta \\
& +\int_{-h_{2}}^{-h_{1}} \int_{t+\theta}^{t} \dot{x}^{T}(s) R_{4} \dot{x}(s) d s d \theta
\end{aligned}
$$

consisting of classical terms used for delay systems ([4], [14], [10]), and an additional term

$$
\begin{aligned}
V_{2, \sigma_{k}}\left(t, x_{t}, \dot{x}_{t}\right) & =\left(t_{k+1}-t\right)\left(\begin{array}{c}
\eta(t) \\
\eta\left(t_{k}\right)
\end{array}\right)^{T} \Omega_{\sigma_{k}}\left(\begin{array}{c}
\eta(t) \\
\eta\left(t_{k}\right)
\end{array}\right) \\
& +\left(t_{k+1}-t\right) \int_{t_{k}}^{t} \dot{\eta}^{T}(s) U_{\sigma_{k}} \dot{\eta}(s) d s \\
& +\left(t_{k+1}-t\right)\left(t-t_{k}\right) \eta^{T}\left(t_{k}\right) S_{\sigma_{k}} \eta\left(t_{k}\right)
\end{aligned}
$$

with the vector $\eta(t)$ :

$$
\eta(t)=\left(\begin{array}{c}
x(t) \\
x(t-h(t))
\end{array}\right),
$$

and the matrices $\Omega_{\sigma}, \sigma \in\{1, \cdots, q\}$ defined as:

$$
\Omega_{\sigma}=\left(\begin{array}{cc}
\frac{X_{\sigma}+X_{\sigma}^{T}}{2} & -X_{\sigma}+X_{1, \sigma} \\
* & -X_{1, \sigma}-X_{1, \sigma}^{T}+\frac{X_{\sigma}+X_{\sigma}^{T}}{2}
\end{array}\right) .
$$

The matrices $P, Q_{1}, Q_{2}, Q_{3}, R_{1}, R_{2}, R_{3}, R_{4}, U_{\sigma}, S_{\sigma}$, $X_{\sigma}, X_{1, \sigma}$ have appropriate dimensions. Parameter $\sigma_{k}$ can be any element $\sigma \in\{1, \cdots, q\}$ such that $x\left(s_{k}\right) \in \mathcal{R}_{\sigma}$ and $\tau^{-} \leq \tau_{k} \leq \tau_{\sigma}^{+}$(there exists at least one, according to (3) and (5)). 
Similar to what we had with the previous simple LKF, we note that the term (26) is composed of matrix terms $\Omega_{\sigma_{k}}$, $U_{\sigma_{k}}$, and $S_{\sigma_{k}}$ which are switching at times $t_{k}$ according to the region $x\left(s_{k}\right)$ belongs to. This state-dependent switch is possible thanks to the fact that $V_{2, \sigma_{k}}\left(t_{k}, x_{t_{k}}, \dot{x}_{t_{k}}\right)=$ $\lim _{t \rightarrow t_{k}^{-}} V_{2, \sigma_{k-1}}\left(t, x_{t}, \dot{x}_{t}\right)=0$, which ensures the continuity of $V_{2}$. This function with state-dependent matrices is a natural extension of the works with LKFs on systems with delays ([4], [14]), sampling ([6], [15]), or both ([10]).

As in the simplified case, we analyse the system's $\mathcal{L}_{2}$ stability by checking the conditions of Lemma 4 with

$$
\bar{V}(t)=V_{\sigma_{k}}\left(t, x_{t}, \dot{x}_{t}\right) \text {, for all } t \in\left[t_{k}, t_{k+1}\right) \text { and } k \in \mathbb{N} \text {, }
$$

with $V_{\sigma_{k}}$ defined in (24). In the following, we also define the time-dependent functions $\bar{V}_{1}$ and $\bar{V}_{2}$, with similar notations

The $\mathcal{L}_{2}$ stability analysis is divided into two main steps. - First, we prove that $\bar{V}$ is continuous over $\mathbb{R}^{+}$and differentiable for all $t \in\left[t_{k}, t_{k+1}\right)$, and provide conditions for its positive definiteness.

- Then, we differentiate $\bar{V}$, upper-bound the obtained result and derive the $\mathcal{L}_{2}$ stability conditions.

We introduce two scalars, to be involved in the next lemma:

$$
\begin{aligned}
& T_{1, \sigma}=T_{1}=\max \left\{\tau^{-}+h_{1}-h_{2}, \frac{\tau^{-}}{1-e_{1}}\right\}, \\
& T_{2, \sigma}=\min \left\{\tau_{\sigma}^{+}+h_{2}-h_{1}, \frac{\tau_{\sigma}^{+}}{1-e_{2}}\right\} .
\end{aligned}
$$

As it is shown in the proof of the lemma (provided in the Technical Report [3]), these scalars are set to satisfy

$$
T_{1, \sigma_{k}} \leq t_{k+1}-t_{k} \leq T_{2, \sigma_{k}}
$$

Lemma 2: The function $\bar{V}$ defined in (29) is continuous over $\mathbb{R}^{+}$and differentiable for all $t \neq t_{k}, k \in \mathbb{N}$. If its matrix parameters satisfy $P \in S_{2 n}^{+*}, Q_{1}, Q_{2}, Q_{3}, R_{1}, R_{2}$, $R_{3}, R_{4} \in S_{n}^{+}, U_{\sigma}, S_{\sigma} \in S_{2 n}^{+}, X_{\sigma}, X_{1, \sigma} \in \mathcal{M}_{2 n, 2 n}$, and if there exist $q$ scalars $\varepsilon_{\sigma} \geq 0$ such that, for all $\sigma \in\{1, \cdots, q\}$ :

$$
\left(\begin{array}{cc}
P & 0 \\
0 & 0
\end{array}\right)+T_{2, \sigma} \Omega_{\sigma}-\varepsilon_{\sigma}\left(\begin{array}{cc}
0 & 0 \\
0 & \Psi_{\sigma}
\end{array}\right) \succ 0,
$$

then $\bar{V}$ is also positive definite, and there exists a scalar $\beta>0$ such that $\bar{V}(t) \geq \beta\|x(t)\|_{2}^{2}$ for all $t \geq 0$.

Proof: The proof is available in the Technical Report [3].

Now that smoothness and positive-definiteness of $\bar{V}$ can be checked, the second step is the stability analysis. We introduce the matrices $M_{i \in\{1, \cdots, 11\}} \in \mathcal{M}_{n, 11 n+n_{w}}$ and $M_{12} \in \mathcal{M}_{n_{w}, 11 n+n_{w}}$ :

$$
\left(\begin{array}{lll}
M_{1}^{T} & \cdots & M_{12}^{T}
\end{array}\right)=I,
$$

and define the matrices $N_{j \in\{1, \cdots, 7\}}$ :

$$
\begin{aligned}
& N_{1}=A M_{1}-B K M_{4}+E M_{12}, N_{2}=\left(\begin{array}{l}
M_{1} \\
M_{2}
\end{array}\right), \\
& N_{3}=\left(\begin{array}{l}
N_{1} \\
M_{7}
\end{array}\right), N_{4}=\left(\begin{array}{l}
M_{3} \\
M_{4}
\end{array}\right), N_{5}=\left(\begin{array}{l}
N_{2} \\
N_{4}
\end{array}\right), \\
& N_{6}=\left(\begin{array}{l}
M_{8} \\
M_{9}
\end{array}\right), \text { and } N_{7}=C M_{1}-D K M_{4} .
\end{aligned}
$$

The use of these matrices is very similar to the one in the previous simplified case (see (16)).

In the following Theorem, we provide an $\mathcal{L}_{2}$-stability condition for the system $\mathbf{T}$. The condition is in the form of LMIs of the size $\left(11 n+n_{w}\right) \times\left(11 n+n_{w}\right)$ that depend on the matrices of the LKF (24), of the description of the conic regions (4), and of some scalars $\left(\varepsilon_{\sigma}, \varepsilon_{i, j, l, o, \sigma}\right)$ and matrices $\left(Y_{1, \sigma}, Y_{2, \sigma}, Y_{3, \sigma}\right)$ resulting from the use of the S-procedure [1] and Finsler's Lemma [1] respectively.

Theorem 3: Consider scalars $\gamma \geq 0, h_{1}, h_{2}, e_{1}, e_{2}$, $\tau^{-}$, and a set of $q$ conic regions covering the state space $\mathcal{R}_{\sigma}=\left\{x, x^{T} \Psi_{\sigma} x \geq 0\right\}, \Psi_{\sigma} \in S_{n}, \sigma \in\{1, \cdots, q\}$, with maximal sampling intervals $\tau_{\sigma}^{+}$.

The perturbed and delayed sampled-data system $\mathbf{T}$ is finitegain $\mathcal{L}_{2}$-stable from $w$ to $z$ with a gain less than $\gamma$ if there exist matrices $P \in S_{2 n}^{+*}, Q_{1}, Q_{2}, Q_{3}, R_{1}, R_{2}, R_{3}, R_{4} \in S_{n}^{+}$, $U_{\sigma}, S_{\sigma} \in S_{2 n}^{+}, X_{\sigma}, X_{1, \sigma} \in \mathcal{M}_{2 n, 2 n}, Y_{1, \sigma} \in \mathcal{M}_{7 n, 2 n}$, $Y_{2, \sigma}, Y_{3, \sigma} \in \mathcal{M}_{7 n, n}$ and scalars $\varepsilon_{\sigma}, \varepsilon_{i, j, l, o, \sigma} \geq 0$ such that (32) and (35) are satisfied for all $\sigma \in\{1, \cdots, q\}$ and $(i, j, l, o) \in\{1,2\}^{4}$ :

$$
\Xi_{i, j, l, o, \sigma}+\varepsilon_{i, j, l, o, \sigma} M_{4}^{T} \Psi_{\sigma} M_{4} \preceq 0,
$$

with

$$
\begin{aligned}
& \Xi_{i, j, l, 1, \sigma}=\Xi_{i, j, \sigma}+T_{l, \sigma}\left[N_{4}^{T} S_{\sigma} N_{4}+N_{3}^{T} U_{\sigma} N_{3}\right. \\
& \left.+N_{3}^{T} \Omega_{1, \sigma} N_{5}+N_{5}^{T} \Omega_{1, \sigma}^{T} N_{3}\right] \text {, } \\
& \Xi_{i, j, l, 2, \sigma}=\Xi_{i, j, \sigma}+T_{l, \sigma}\left[-N_{4}^{T} S_{\sigma} N_{4}-N_{6}^{T} U_{\sigma} N_{6}\right. \\
& \left.+\bar{Y}_{1, \sigma} N_{6}+N_{6}^{T} \bar{Y}_{1, \sigma}^{T}\right] \text {, } \\
& \Xi_{i, j, \sigma}=N_{3}^{T} P N_{2}+N_{2}^{T} P N_{3}+M_{1}^{T} Q_{1} M_{1} \\
& +M_{5}^{T}\left(Q_{2}-Q_{1}\right) M_{5}-M_{6}^{T} Q_{3} M_{6}-N_{5}^{T} \Omega_{\sigma} N_{5} \\
& +N_{1}^{T}\left(R_{1}+h_{j} R_{2}+h_{2} R_{3}+\left(h_{2}-h_{1}\right) R_{4}\right) N_{1} \\
& -\frac{1}{1-e_{1}} M_{7}^{T} R_{1} M_{7}+\left(1-e_{i}\right) M_{2}^{T}\left(Q_{3}-Q_{2}\right) M_{2} \\
& -\frac{1}{h_{1}}\left(M_{1}-M_{5}\right)^{T}\left(\left(1-e_{i}\right) R_{2}+R_{3}\right)\left(M_{1}-M_{5}\right) \\
& -\left(h_{j}-h_{1}\right) M_{10}^{T}\left(\left(1-e_{2}\right) R_{2}+R_{3}+R_{4}\right) M_{10} \\
& -\left(h_{2}-h_{j}\right) M_{11}^{T}\left(R_{3}+R_{4}\right) M_{11} \\
& +\bar{Y}_{1, \sigma}\left(-N_{2}+N_{4}\right)+\left(-N_{2}+N_{4}\right)^{T} \bar{Y}_{1, \sigma}^{T} \\
& +\bar{Y}_{2, \sigma}\left(\left(h_{j}-h_{1}\right) M_{10}-M_{5}+M_{2}\right) \\
& +\left(\left(h_{j}-h_{1}\right) M_{10}-M_{5}+M_{2}\right)^{T} \bar{Y}_{2, \sigma}^{T} \\
& +\bar{Y}_{3, \sigma}\left(\left(h_{2}-h_{j}\right) M_{11}-M_{2}+M_{6}\right) \\
& +\left(\left(h_{2}-h_{j}\right) M_{11}-M_{2}+M_{6}\right)^{T} \bar{Y}_{3, \sigma}^{T} \\
& +N_{7}^{T} N_{7}-\gamma^{2} M_{12}^{T} M_{12} \text {, } \\
& \Omega_{1, \sigma}=\left(\begin{array}{cc}
\frac{X_{\sigma}+X_{\sigma}^{T}}{2} & -X_{\sigma}+X_{1, \sigma}
\end{array}\right), \\
& \bar{Y}_{1, \sigma}=\left(\begin{array}{c}
Y_{1, \sigma} \\
0
\end{array}\right) \in \mathcal{M}_{11 n+n_{w}, 2 n} \\
& \bar{Y}_{a, \sigma}=\left(\begin{array}{c}
Y_{a, \sigma} \\
0
\end{array}\right) \in \mathcal{M}_{11 n+n_{w}, n}, a \in\{2,3\} \text {. }
\end{aligned}
$$


Proof: The proof, similar to the one in the non-delayed case, is available in the Technical Report [3].

Remark 1: If $w$ satisfies $z^{T}(t) z(t)-\gamma^{2} w^{T}(t) w(t) \geq 0$, and if the LMIs (35) are strict, the sampled-data system $\mathbf{T}$ is asymptotically stable.

\section{A STATE-DEPENDENT SAMPLING MAP DESIGN}

In this section, we propose a three-step algorithm (Figure 1), which allows for computing the LKF matrices so as to maximize both the largest admissible state-independent sampling interval $\tau^{+}=\min _{\sigma \in\{1, \cdots, q\}} \tau_{\sigma}^{+}$and the statedependent sampling map $\tau_{\max }$ described in (5), with respect to the $\mathcal{L}_{2}$-stability conditions provided in Theorem 3 . Keep in mind that all steps are made offline.

\section{STEP 1}

\begin{tabular}{|c|}
\hline \multicolumn{2}{|c|}{$\diamond$ Single region $\mathbb{R}^{n}$} \\
Theorem 3 LMIs $+\quad$ line search algorithm \\
\hline$\diamond$ Compute the largest admissible \\
state-independent sampling $\tau^{+}$ \\
$\diamond$ Compute the LKF matrices $P, Q_{1}, Q_{2}, Q_{3}$, \\
$R_{1}, R_{2}, R_{3}, R_{4}$, (and $\left.U, S, X, X_{1}\right)$
\end{tabular}

\section{STEP 2}

$\diamond q$ conic regions $\mathcal{R}_{\sigma}$ covering the state space $\diamond$ Use the LKF matrices $P, Q_{1}, Q_{2}, Q_{3}$, $R_{1}, R_{2}, R_{3}, R_{4}$ computed in Step 1

Theorem 3 LMIs $+\quad \begin{gathered}\text { line search algorithm } \\ \text { for each region } \mathcal{R}_{\sigma}\end{gathered}$

$\diamond$ Design the mapping of the largest admissible sampling $\tau_{\sigma}^{+} \geq \tau^{+}$for each region $\mathcal{R}_{\sigma}$

$\diamond$ Compute the LKF matrices $U_{\sigma}, S_{\sigma}, X_{\sigma}, X_{1, \sigma}$ for each region $\mathcal{R}_{\sigma}$

\section{STEP 3}

Design of the state-dependent sampling map $\tau_{\max }(x)=\max _{\sigma \in \mathcal{I}(x)} \tau_{\sigma}^{+} \geq \tau^{+}, \forall x \in \mathbb{R}^{n}$

Fig. 1. Algorithm to design the state-dependent sampling map $\tau_{\max }$

Remark 2: Step 1 may be used as a robust analysis of delayed and perturbed sampled-data systems with timevarying sampling. Step 2 leads to a self-triggering algorithm except that all computations are made offline, and the state- dependent matrices $U_{\sigma}, S_{\sigma}, X_{\sigma}$, and $X_{1, \sigma}$ of the LKF are computed during that step.

Remark 3: One can also compute the largest admissible state-independent sampling $\tau^{+}$(Step 1) by using the LKF with state-dependent matrices switching on some predefined regions $\mathcal{R}_{\sigma}$. Although it is more complex, this can reduce the conservatism, as it will be illustrated in Example 2.

Remark 4: Very often, the works are carried with a minimal sampling interval $\tau^{-}$set to 0 , as in [2], [17], or [6]. Enabling a larger minimal sampling makes it possible to increase the obtained maximal sampling $\tau_{\sigma}^{+}$(or even $\tau^{+}$) with the proposed technique.

\section{NUMERICAL EXAMPLES}

\section{A. Example 1}

We consider the system:

$$
\begin{aligned}
& \dot{x}(t)=\left(\begin{array}{cc}
-3 & 0 \\
0 & 1
\end{array}\right) x(t)-\left(\begin{array}{l}
1 \\
1
\end{array}\right) K x\left(s_{k}\right)+w(t), \text { and } \\
& K=\left(\begin{array}{ll}
-1 & 4
\end{array}\right), z(t)=x(t), \text { for } t \in\left[t_{k}, t_{k+1}\right) .
\end{aligned}
$$

The state-dependent sampling map (5) will be designed in four successive cases: 1$)$ no delay nor perturbations ( $w=$ $0, h=0$, asymptotic stability);2) perturbations on the delayfree system $\left(w \neq 0, h=0, \mathcal{L}_{2}\right.$-stability with $\left.\gamma=\sqrt{10}\right)$; 3) unperturbed system with delay $h(t) \in\left[10^{-4}, 10^{-1}\right]$ and $\dot{h}(t) \in[-0.2,0.6]$ ( $w=0, h \neq 0$, asymptotic); 4) perturbed system with the same delay $(w \neq 0, h \neq 0, \gamma=\sqrt{10})$.

We set $\tau^{-} \simeq 0$ and use the isotropic partition described in [2] to design a covering of $q=100$ conic regions. Then, we use the algorithm of Section IV to build the mapping that maximizes the sampling interval for each state. Because the state dimension is 2 , the conic regions are defined from the spherical coordinates $(\rho, \theta)$ of the state $x=\rho \mathrm{e}^{\mathrm{i} \theta}$, for the particular value $\rho=1$ (the unit sphere). Computed offline in each of the 4 cases, Figure 2 presents the admissible sampling interval as a function of the state angle $\theta \in[-\pi, \pi)$.

Respectively to the four presented cases, the longest stateindependent sampling interval we found is: 1) $\tau^{+}=0.535$; 2) 0.445 ; 3) 0.169 ; 4) 0.145 . Note that since $\tau^{-} \simeq 0$, the system $\mathcal{L}_{2}$-stability (or asymptotic stability) is preserved for any time-varying sampling less than $\tau^{+}$. Also, thanks to the mappings we built, the robustness regarding classical timevarying sampling is extended to all state-dependent sampling intervals under the curves obtained in Figure 2.

Simulation results are shown in the Technical Report [3].

\section{B. Example 2}

To show the conservatism reduction brought by the LKF with state-dependent matrices, we consider the unperturbed, delay-free system from [9]:

$$
\begin{aligned}
& \dot{x}(t)=\left(\begin{array}{cc}
-0.5 & 0 \\
0 & 3.5
\end{array}\right) x(t)-\left(\begin{array}{l}
1 \\
1
\end{array}\right) K x\left(s_{k}\right), \text { and } \\
& K=\left(\begin{array}{ll}
-1.02 & 5.62
\end{array}\right), z(t)=x(t), \text { for } t \in\left[s_{k}, s_{k+1}\right) .
\end{aligned}
$$



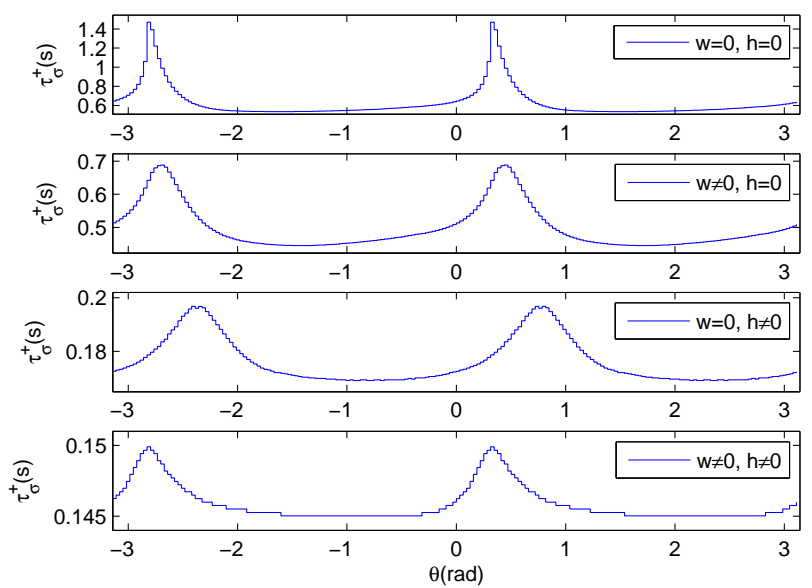

Fig. 2. Example 1: Mapping of the maximal admissible samplings $\tau_{\sigma}^{+}$for the system with or without perturbations $w$ and/or delays $h$.

We set $\tau^{-} \simeq 0$. Considering the results given by the step 1 of the algorithm described in Section IV and taking only one region $\mathbb{R}^{n}$, the longest state-independent sampling interval $\tau^{+}$(i.e. admissible no matter the state) obtained is equal to 0.267 , whereas we obtain 0.309 with $q=100$ regions $\mathcal{R}_{\sigma}$ (an improvement of $16 \%$ ). This corresponds to a robust stability bound that can be compared to the ones obtained in [6] $\left(\tau^{+}=0.259\right)$, [7] $\left(\tau^{+}=0.204\right)$, or [15] $\left(\tau^{+}=0.198\right)$.

\section{CONCLUSION}

This work introduced a new class of Lyapunov-Krasovskii functionals with state-dependent matrices. It allowed for designing a state-dependent sampling that reduces the number of actuations, while keeping the $\mathcal{L}_{2}$-stability for perturbed time-delayed linear state feedback systems. The proposed method can be seen both as a self-triggered control and as a new time-varying sampling analysis leading to a state-dependent sampling design. We think it presents two main advantages, since it makes it possible:

- to maximize the minimal sampling interval $\tau^{+}=\inf _{x \in \mathbb{R}^{n}} \tau_{\max }(x)$ of the state-dependent sampling map, and to compute the associated Lyapunov-Krasovskii function matrices that ensure the system $\mathcal{L}_{2}$-stability;

- to design offline a mapping of the state space with a maximum allowable sampling time for each region. Therefore, no additional computation is required online during the control of the system.

\section{APPENDIX}

Lemma 4: (Adapted from [6]) $\mathbf{T}$ is $\mathcal{L}_{2}$-stable from $w$ to $z$ with a gain less than $\gamma \geq 0$ if there exists a positive definite continuous function $\bar{V}: t \in \mathbb{R}^{+} \rightarrow \bar{V}(t) \in \mathbb{R}^{+}$, differentiable for all $t \neq t_{k}, k \in \mathbb{N}$, that satisfies along $\mathbf{T}$ :

$$
\dot{\bar{V}}(t)+z^{T}(t) z(t)-\gamma^{2} w^{T}(t) w(t) \leq 0 .
$$

Lemma 5: (Adapted from [1]) Consider $x \in \mathbb{R}^{n}$, two matrices $\Gamma_{1}$ and $\Gamma_{2}$ in $S_{n}$ and two scalars $\lambda^{-}<\lambda^{+}$. The following statements are equivalent:

(i) $\forall \lambda \in\left[\lambda^{-}, \lambda^{+}\right], x^{T}\left(\Gamma_{1}+\lambda \Gamma_{2}\right) x \leq 0$,

(ii) $x^{T}\left(\Gamma_{1}+\lambda^{-} \Gamma_{2}\right) x \leq 0$ and $x^{T}\left(\Gamma_{1}+\lambda^{+} \Gamma_{2}\right) x \leq 0$.

\section{REFERENCES}

[1] S. Boyd, L. El-Ghaoui, E. Feron, and V. Balakrishnan, "Linear Matrix Inequalities in System and Control Theory", in SIAM, Studies in Applied Mathematics, 15, pp. 23-24, 1994.

[2] C. Fiter, L. Hetel, W. Perruquetti, and J.-P. Richard, "A State Dependent Sampling for Linear State Feedback", in Automatica, 48(8), pp. 1860-1867, 2012. doi: 10.1016/j.automatica.2012.05.063

[3] C. Fiter, L. Hetel, W. Perruquetti, and J.-P. Richard, "StateDependent Sampling for LTI Systems with Perturbations and Delays", in Technical Report, LAGIS, Available at http://docs.google.com/open?id=0B0wsy2L71luCQVFZVFVIU2F3a3c

[4] E. Fridman, "New Lyapunov-Krasovskii Functionals for Stability of Linear Retarded and Neutral Type Systems", in Systems and Control Letters, 43(4), pp. 309-319, 2001.

[5] E. Fridman, A. Seuret, and J.-P Richard, "Robust Sampled-Data Stabilization of Linear Systems: An Input Delay Approach", in Automatica, 40(8), pp.1441-1446, 2004

[6] E. Fridman, "A Refined Input Delay Approach to Sampled-Data Control", in Automatica, 46(2), pp. 421-427, 2010.

[7] H. Fujioka, "Stability Analysis of Systems with Aperiodic Sampleand-Hold Devices", in Automatica, 45(3), pp. 771-775, 2009.

[8] K. Gu, V. Kharitonov, and J. Chen, "Stability of Time-Delay Systems", in Boston: Birkhuser, 2003.

[9] L. Hetel, A. Kruszewski, W. Perruquetti, and J.-P Richard, 'Discrete and Intersample Analysis of Systems with Aperiodic Sampling", in IEEE Transactions on Automatic Control, 56(7), pp. 1696-1701, 2011.

[10] W. Jiang and A. Seuret, "Improved Stability Analysis of Networked Control Systems Under Asynchronous Sampling and Input Delay", in 2nd IFAC Workshop on Distributed Estimation and Control in Networked Systems, Annecy, France, 2010.

[11] K. Liu and E. Fridman, "Stability Analysis of Networked Control Systems: A Discontiuous Lyapunov Functional Approach", in 48th IEEE Conference on Decision and Control, Shanghai, China, 2009.

[12] J. Lunze and D. Lehmann, "A State-Feedback Approach to EventBased Control", in Automatica, 46(1), pp. 211-215, 2010.

[13] M. Mazo-Jr., A. Anta, and P. Tabuada, "An ISS Self-Triggered Implementation of Linear Controllers", in Automatica, 46(8), pp. 13101314,2010

[14] J.-P Richard, "Time Delay Systems: an Overview of Some Recent Advances and Open Problems", in Automatica, 39(10), pp. 1667-1694, 2003

[15] A. Seuret, "Stability Analysis for Sampled-Data Systems with a TimeVarying Period", in 48th IEEE Conference on Decision and Control, Shanghai, China, 2009.

[16] P. Tabuada, "Event-Triggered Real-Time Scheduling of Stabilizing Control Tasks", in IEEE Transactions on Automatic Control, 52(9), pp. 1680-1685, 2007.

[17] X. Wang and M. Lemmon, "Self-Triggering Under State-Independent Disturbances", in IEEE Transactions on Automatic Control, 55(6), pp. 1494-1500, 2010. 